\title{
EXAMINING THE IMPACT OF SOCIAL MEDIA ON THE ACADEMIC PERFORMANCES OF SAUDI STUDENTS - CASE STUDY: PRINCE SATTAM BIN ABDUL AZIZ UNIVERSITY
}

Syed Nasrullah ${ }^{1 *}$, M. Firdouse Rahman Khan ${ }^{2}$

${ }^{1}$ Department of Computer, Community College in Al-Kharj, Prince Sattam bin Abdulaziz University, Al-Kharj 11942, Saudi

Arabia, ${ }^{2}$ Faculty of Business, Sohar University, Oman.

Email: ${ }^{1 *}$ nasrullah4@gmail.com, ${ }^{2}$ Firdouse4u@yahoo.co.uk

Article History: Received on $24^{\text {th }}$ August 2019, Revised on $25^{\text {th }}$ September 2019, Published on $29^{\text {th }}$ October 2019

\begin{abstract}
Purpose: The objective of the research study is to investigate whether there is a positive impact of the use of social media on undergraduate students' academic performances and their social interpersonal skills in the Prince Sattam bin Abdulaziz University.
\end{abstract}

Design/methodology/approach: 64 samples were collected from full-time undergraduate students studying in Prince Sattam bin Abdulaziz University from different colleges including Community college, College of Business Administration, College of Education, College of Engineering and College of Pharmacy. The descriptive statistics analysis was used to analyze the demographic data while inferential statistics were used in testing the research hypotheses. The results obtained from the analyses were used to interpret the outcomes.

Findings: The empirical results reveal that the students enjoy meeting new friends online using social media rather than meeting in person and for this reason they spend a lot of time - addicted. It is also confirmed that the bad comments are passed easily through social media affecting other's sentiments and most of them strongly believe that all the information in social media is true and reliable and the rumors were spread easily in social media. Further, it is also confirmed that social media does not support the Learning of the students.

Practical Implications: The study confirms that the students exchange learning materials through social media and it helps them to update the developments in their college/university. The students believe that through Social media they have improved their communication skills and they can communicate with anyone at any time. It is also found that some of their teachers communicate with them and encourage them to use social media but for studying only.

Originality/value: The research work is of its first kind as it focuses on the impact of social media on the academic performances of the students studying in Prince Sattam bin Abdulaziz University, Saudi Arabia, which has suggested effective means for effective implementation of social media strategy.

Keywords: Social Media Networks, Saudi Arabian Students, Academic Performance, Interpersonal skills, Social Media Addiction.

\section{INTRODUCTION}

Social Media has become one of the most important communication means in recent times towards the exchange of knowledge, data and the unknown information. Social media is a form of electronic communication through which people interact with each other. Social media has attracted millions of internet users, who have integrated the social networking sites (SNS) in their daily lives routines, as a powerful medium in enhancing the communication between the society on the whole. Social media has brought radical changes in society creating both positive and negative impacts. Any information whether good or bad can be easily spread over through social media. As Social Media Technology (SMT) is growing fast, as is not only restricted to net-based technology but also compatible with mobile technology, it convenes any user to complete his/her communication easily through their digital devices. It is confirmed that Twitter, Whatsapp, Instagram and Facebook as the most popular social media platforms (Alwagait, Shahzad, \& Alim, 2015; Michikyan, Subrahmanyam, \& Dennis, 2015).

Khan, Hatami, Sasidharan, and Al-Roshdi (2017) found that people are actively participating in one or the other social media platforms and reap benefits out of it. Baker (2009) advocated that the use of social networks could be an effective tool to motivate people to develop future bonding. SNS has become nodal points for the youth to convey their thoughts in unique ways with other like-minded people and considered as a place to share their artistic abilities (Boyd, 2007b). Through the socalled blogs of such networking sites, people get prior information about the institutions to which they are willing to join, etc.

Social Media has also totally changed students' way of learning and their studying style and the overall set-up. Students started using these sites as resources to obtain information and facilitate themselves towards their completing assessments, projects, and related academic work (El Khatib \& Khan, 2017). University students make up the major proportion of the online networking community. In addition, given the popularity of social media, many professors have started to use social media for 
enhancing communication with and among students in their classes, class discussions, and teamwork on projects to improve learning outcomes. Many Higher Education Institutions (HEIs) have even started using web technology in promoting education and have started implementing their assessments using such growing technologies. Hence, the level of understanding of the students is becoming high and their learning expectations also becoming high. This is triggering social media to become a catalyst in the field of education growth.

Early studies have proven the effect of rapid and heavy communication technology used by students on their academic performances (Ellore, Niranjan, \& Brown, 2014; Junco, 2015; Lomi, Snijders, Steglich, \& Torló, 2011; Mehmood \& Taswir, 2013). However, there are mixed opinions on the effect of the impact of social media on the academic performances of the students at different levels.

Social media network sites can have a positive or negative impact on students' academic performance. Many argue on the risks involved in using SNS whereas it has its own benefits. They fear that the students' addiction to social media can negatively affect students' academic performances. Some researchers claim a little or no negative effects on students' academic performance if good multitasking is achieved by students. The rising impact of social media on the private and working lives of students has made researchers and educational institutions to study and rethink the concept and content of educational development in the digital age.

Therefore, our study aims to add better clarity to this research area by examining the relationship between the use of social media and Saudi students' academic performances. Thus, the prime objective of the research study is to investigate whether there is a positive impact of the use of social media on undergraduate students' academic performances and their social interpersonal skills in the Prince Sattam bin Abdulaziz University.

Although many research results pointed to a negative impact of social media usage on academic performance (Paul, Baker, \& Cochran, 2012; Wentworth \& Middleton, 2014).

\section{REVIEW OF LITERATURE}

Paul et al. (2012) suggested that online social networks could possibly be viewed as helpful educational technology if the more academic staff actually knew how to incorporate them into their curricula. Amin, Mansoor, Hussain, and Hashmat (2016) claimed that social media networking sites draw the attention of the students and increase their academic grade points. Mingle and Adams (2015) insisted that social media should be used for educational purposes but with proper monitoring and counseling as the chances of students becoming addicted so social media is high. Butler and Matook (2015) confirmed that social media affect the relationships of the people and the methods of their learning. Bankers also use social media sites to enhance customer service by means of building a relationship through Electronic Customer Relations Management depending upon the information reliability and responsiveness (Khan, Al-balushi, Algaithi, \& Al-Shihi, 2017). Chretien, Farnan, Greysen, and Kind (2011) confirmed that internal medicine educators are using SNS and interacting with their trainees online. Allam and Elyas (2016) indicated that the majority of the academia believe strongly in the pedagogical values and benefits of using social media as an English language training tool in the Saudi context but they expressed reservations using in the classrooms.

Osharive (2015) propounded that students should be motivated to use social for educational purposes and the networking sites should expand folios for academic activities. Alsuraihi, Almaqati, Abughanim, and Jastaniah (2016) suggested the utilization of social media in education by developing intrinsic activities as it is beneficial. Utilizing social media tools in education sometimes can be very demanding and challenging for educators to assure effective learning (Yusuf, AL-Madah, \& Alam, 2016). Paul et al. (2012) indicated that the students' academic performance is a function of devotion span, time management skills, student characteristics, and time spent on online social media. Owusu-Acheaw and Larson (2015) insisted that students should be enthused to use handphones with internet facilities but with restricted timing to social media as it adversely affects their academic achievements.

Masters (2015) claimed that the students are becoming highly addicted to and intermediation is needed to control the same and also reported that YouTube, Facebook, and Twitter were the most used social media platforms (in their order of preference). Ali Aljabry et al. (2017) proved that the most common social media platform used was Facebook and social media had affected the academic performance of students negatively. Jameel et al. (2019) claimed that male students are spending more time on social media and WhatsApp and Facebook as their favorite platforms. El-Badawy and Hashem (2015) advocated that there exists no relationship between social media and the student's academic performance. But, Amadi and Ewa (2018) confirmed that the social media platforms had distracted the attention of the students from their studies resulting in negative performances. Rouis, Limayem, and Salehi-Sangari (2011a) indicated that extensive use of Facebook by students with extraverted personalities leading to poor academic performances. Hasnain, Nasreen, and Ijaz (2015) proved that social media has an inverse relationship with academic performance due to fading communication barriers and rising easy access in data. Halboub et al. (2016) found out that the majority of students are using their smartphones/computers for social networks on a 
daily basis, and during lectures, laboratories, and clinics as well, resulting in negative performance and the only suggested remedy is to stop or reduce using social media.

Greysen, Kind, and Chretien (2010) confirmed that the rise of social media content created by internet users and hosted by popular sites such as Facebook, Twitter, YouTube, and Wikipedia, and blogs have brought several new hazards in medical education. Al-Tarawneh (2014) claimed that social media influences the students badly in the form of addiction, overloaded information, wastage of time, and physical isolation from society. A study using the Bergen Facebook addiction scale confirmed that health science students were highly addicted to social networking websites (Squmedia-ar, 2015). Boyd (2007a) claimed that the current generation youth are spending a great deal of time in SNS like MySpace, Facebook, and Bebo, to access public life. Social media use harming sleep quality, self-esteem, and mental health and affect academic performances in medical students (Al Suwayri, 2016). Griffiths (2013) opined that students may be compelled to retain their online connections leading to excessive usage of social media. Yet, time management is the factor that contributes to negative academic performance besides excessive social media use (Karpinski, Kirschner, Ozer, Mellott, \& Ochwo, 2013).

\section{RESEARCH METHODOLOGY}

After going through the above literature review, and a well-defined questionnaire was prepared to collect the data on similar lines of research questionnaire used in Oman (El Khatib \& Khan, 2017). 64 samples were collected from the students studying in various colleges of Prince Sattam bin Abdul Aziz University viz. Community college, College of Business Administration, College of Education, College of Engineering and College of Pharmacy. The collected data was compiled, tabulated and analyzed using SPSS. The descriptive statistics analysis was used to analyze the demographic data whereas inferential statistics were carried out in testing the research hypotheses. The results thus obtained were used to interpret the outcomes, and the suggestions were drawn.

\section{FINDINGS}

Table1: Reliability Analysis of the data

\begin{tabular}{lll}
\hline & $\mathbf{N}$ & $\mathbf{\%}$ \\
\hline Valid Cases & 64 & 100.0 \\
Excluded & 0 & 0.0 \\
\hline
\end{tabular}

\begin{tabular}{cc}
\hline Cronbach's Alpha & N of items \\
\hline .860 & 43 items \\
\hline
\end{tabular}

The test for data reliability and internal consistency confirms that the value lies in between $.70 \& .93$.

Table 2: Demographic details of the respondents

\begin{tabular}{llcc}
\hline \multicolumn{1}{c}{ Characteristics } & & Frequency & $\%$ \\
\hline \multirow{2}{*}{ Nationality } & Saudi & 64 & 100.0 \\
\cline { 2 - 4 } & Expatriate & 0 & 0.0 \\
\hline \multirow{2}{*}{ Gender } & Male & 40 & 62.5 \\
\cline { 2 - 4 } & Female & 24 & 37.5 \\
\hline \multirow{3}{*}{ Age } & $18-<20$ years & 25 & 39.1 \\
\cline { 2 - 4 } & $20-<23$ years & 36 & 56.3 \\
\cline { 2 - 4 } & $23-<25$ years & 3 & 4.7 \\
\hline \multirow{2}{*}{ Marital Status } & Single & 64 & 100.0 \\
\cline { 2 - 4 } & Married & 0 & 0.0 \\
\hline \multirow{5}{*}{ College } & Community College & 30 & 46.9 \\
\cline { 2 - 4 } & College of Business Administration & 2 & 3.1 \\
\cline { 2 - 4 } & College of Education & 8 & 12.5 \\
\cline { 2 - 4 } & College of Engineering & 8 & 12.5 \\
\cline { 2 - 4 } & College of Pharmacy & 16 & 25.0 \\
\hline \multirow{4}{*}{ Qualification Level } & Level 1 & 16 & 25.0 \\
\cline { 2 - 4 } & Level 2 & 40 & 62.5 \\
\cline { 2 - 4 } & Level 3 & 8 & 12.0 \\
\cline { 2 - 4 } & Level 4 & 9 & 14.1 \\
\hline Employment Status & Working & & \\
\hline
\end{tabular}




\begin{tabular}{|c|c|c|c|}
\hline & Not working & 55 & 85.9 \\
\hline \multirow{4}{*}{$\begin{array}{l}\text { GPA during Last } \\
\text { Semester }\end{array}$} & $<2$ & 5 & 7.8 \\
\hline & $2-3$ & 18 & 28.1 \\
\hline & $3-4$ & 13 & 20.3 \\
\hline & $>4$ & 28 & 43.8 \\
\hline \multirow{4}{*}{ GPA during Last Year } & $<2$ & 7 & 10.9 \\
\hline & $2-3$ & 2 & 3.1 \\
\hline & $3-4$ & 16 & 25.0 \\
\hline & $>4$ & 39 & 60.9 \\
\hline \multirow{3}{*}{ Increase in GPA } & Yes. Increased & 28 & 43.8 \\
\hline & No. Not Increased & 28 & 43.8 \\
\hline & Remains the same & 8 & 12.5 \\
\hline \multirow{7}{*}{ Social Media Used } & Instagram & 3 & 4.7 \\
\hline & WhatsApp & 14 & 21.9 \\
\hline & Twitter & 2 & 3.1 \\
\hline & Instagram \& Whatsapp & 1 & 1.6 \\
\hline & WhatsApp \& Twitter & 1 & 1.6 \\
\hline & Instagram, WhatsApp \& Twitter & 30 & 46.9 \\
\hline & All four & 13 & 20.3 \\
\hline \multirow{7}{*}{$\begin{array}{l}\text { Social Media used for } \\
\text { Educational Purpose }\end{array}$} & Instagram & 0 & 0.0 \\
\hline & WhatsApp & 14 & 21.9 \\
\hline & Twitter & 10 & 15.6 \\
\hline & Instagram \& Whatsapp & 1 & 1.6 \\
\hline & WhatsApp \& Twitter & 4 & 6.3 \\
\hline & Instagram, WhatsApp \& Twitter & 22 & 34.4 \\
\hline & All four & 13 & 20.3 \\
\hline \multirow{6}{*}{$\begin{array}{l}\text { Surfing Time on } \\
\text { Social Media }\end{array}$} & Daily & 36 & 56.3 \\
\hline & Weekly & 0 & 0.0 \\
\hline & Monthly & 0 & 0.0 \\
\hline & Sometimes & 9 & 14.1 \\
\hline & Always & 19 & 29.7 \\
\hline & Never & 0 & 0.0 \\
\hline \multirow{4}{*}{ Daily Surfing Hours } & $<1$ hour & 13 & 20.3 \\
\hline & $1-2$ hours & 3 & 4.7 \\
\hline & $2-4$ hours & 18 & 28.1 \\
\hline & $>4$ hours & 30 & 46.9 \\
\hline
\end{tabular}

Source: Questionnaire

Table 3: Learning

\begin{tabular}{|c|c|c|c|c|c|c|c|c|c|}
\hline$\#$ & Statement & SD & D & $\mathbf{N}$ & $\mathbf{A}$ & SA & $\begin{array}{c}\mathrm{K}-\mathrm{S} \\
\text { value }\end{array}$ & $\begin{array}{c}\text { Chi } \\
\text { Square }\end{array}$ & $\begin{array}{c}P \\
\text { value }\end{array}$ \\
\hline 1 & $\begin{array}{l}\text { I use social media in the } \\
\text { classroom to learn new } \\
\text { information }\end{array}$ & $\begin{array}{c}1 \\
1.6 \%\end{array}$ & $\begin{array}{c}0 \\
0.0 \%\end{array}$ & $\begin{array}{c}8 \\
12.5 \%\end{array}$ & $\begin{array}{c}26 \\
40.6 \%\end{array}$ & $\begin{array}{c}29 \\
45.3 \%\end{array}$ & 0.267 & \multirow{4}{*}{36.875} & \multirow{4}{*}{.000} \\
\hline 2 & $\begin{array}{l}\text { I use social media to update } \\
\text { my academic grades }\end{array}$ & $\begin{array}{c}0 \\
0.0 \%\end{array}$ & $\begin{array}{r}2 \\
3.1 \%\end{array}$ & $\begin{array}{c}19 \\
29.7 \%\end{array}$ & $\begin{array}{c}37 \\
57.8 \%\end{array}$ & $\begin{array}{c}6 \\
9.4 \%\end{array}$ & 0.325 & & \\
\hline 3 & $\begin{array}{l}\text { Social media helps me to } \\
\text { update the developments in } \\
\text { my college / university }\end{array}$ & $\begin{array}{c}2 \\
3.1 \%\end{array}$ & $\begin{array}{c}1 \\
1.6 \%\end{array}$ & $\begin{array}{c}3 \\
4.7 \%\end{array}$ & $\begin{array}{c}42 \\
65.6 \%\end{array}$ & $\begin{array}{c}16 \\
25.0 \%\end{array}$ & 0.367 & & \\
\hline 4 & $\begin{array}{l}\text { My teacher encourages me } \\
\text { to use social media to study }\end{array}$ & $\begin{array}{c}0 \\
0.0 \%\end{array}$ & $\begin{array}{c}0 \\
0.0 \%\end{array}$ & $\begin{array}{c}6 \\
9.4 \%\end{array}$ & $\begin{array}{c}39 \\
60.9 \%\end{array}$ & $\begin{array}{c}19 \\
29.7 \%\end{array}$ & 0.337 & & \\
\hline
\end{tabular}




\begin{tabular}{|c|c|c|c|c|c|c|c|}
\hline 5 & $\begin{array}{l}\text { My classmates exchange } \\
\text { learning materials through } \\
\text { social media }\end{array}$ & $\begin{array}{c}0 \\
0.0 \%\end{array}$ & $\begin{array}{c}0 \\
0.0 \%\end{array}$ & $\begin{array}{c}7 \\
10.9 \%\end{array}$ & $\begin{array}{c}17 \\
26.6 \%\end{array}$ & $\begin{array}{c}40 \\
62.5 \%\end{array}$ & 0.384 \\
\hline 6 & $\begin{array}{l}\text { My learning gets distracted } \\
\text { by the usage of social media }\end{array}$ & $\begin{array}{c}7 \\
10.9 \%\end{array}$ & $\begin{array}{c}7 \\
10.9 \%\end{array}$ & $\begin{array}{c}18 \\
28.1 \%\end{array}$ & $\begin{array}{c}27 \\
42.2 \%\end{array}$ & $\begin{array}{c}5 \\
7.8 \%\end{array}$ & 0.250 \\
\hline 7 & $\begin{array}{l}\text { My study schedule gets } \\
\text { disrupted by the usage of } \\
\text { social media }\end{array}$ & $\begin{array}{c}13 \\
20.3 \%\end{array}$ & $\begin{array}{c}21 \\
32.8 \%\end{array}$ & $\begin{array}{c}15 \\
23.4 \%\end{array}$ & $\begin{array}{c}10 \\
15.6 \%\end{array}$ & $\begin{array}{c}5 \\
7.8 \%\end{array}$ & 0.215 \\
\hline 8 & $\begin{array}{l}\text { I get to learn differently } \\
\text { from diversified students } \\
\text { through social media }\end{array}$ & $\begin{array}{c}4 \\
6.3 \%\end{array}$ & $\begin{array}{c}2 \\
3.1 \%\end{array}$ & $\begin{array}{c}23 \\
35.9 \%\end{array}$ & $\begin{array}{c}26 \\
40.6 \%\end{array}$ & $\begin{array}{c}9 \\
14.1 \%\end{array}$ & 0.229 \\
\hline
\end{tabular}

The above table shows that the p-value is less than 0.05 . So the null hypothesis that 'there is no relationship between the learning of the students and the choices of the respondents' is rejected. i.e., it connotes that there is a significant relationship between the learning of the students and the choices of the respondents. Comparing the K-S values obtained from Kolmogorov-Smirnov test, it is observed that 'My classmates exchange learning materials through social media' ranks first (0.384) followed by 'Social media helps me to update the developments in my college/university' (0.367) and 'My teacher encourages me to use social media to study (0.337).'

Table 4: Communication

\begin{tabular}{|c|c|c|c|c|c|c|c|c|c|}
\hline \# & Statement & SD & D & $\mathbf{N}$ & $\mathbf{A}$ & SA & $\begin{array}{c}\text { K-S } \\
\text { Value }\end{array}$ & $\begin{array}{c}\text { Chi } \\
\text { Square }\end{array}$ & $\begin{array}{c}P \\
\text { value }\end{array}$ \\
\hline 1 & $\begin{array}{l}\text { I have improved my } \\
\text { communication skills } \\
\text { through Social media }\end{array}$ & $\begin{array}{c}0 \\
0.0 \%\end{array}$ & $\begin{array}{c}0 \\
0.0 \%\end{array}$ & $\begin{array}{c}4 \\
6.3 \%\end{array}$ & $\begin{array}{c}50 \\
78.1 \%\end{array}$ & $\begin{array}{c}10 \\
15.6 \%\end{array}$ & 0.424 & \multirow{10}{*}{26.125} & \multirow{10}{*}{.016} \\
\hline 2 & $\begin{array}{l}\text { My English become } \\
\text { worse because of social } \\
\text { media usage }\end{array}$ & $\begin{array}{c}36 \\
56.3 \%\end{array}$ & $\begin{array}{c}16 \\
25.0 \%\end{array}$ & $\begin{array}{r}3 \\
4.7 \%\end{array}$ & $\begin{array}{c}5 \\
7.8 \%\end{array}$ & $\begin{array}{c}4 \\
6.3 \%\end{array}$ & 0.315 & & \\
\hline 3 & $\begin{array}{l}\text { I communicate easily } \\
\text { with my classmates } \\
\text { through social media }\end{array}$ & $\begin{array}{c}0 \\
0.0 \%\end{array}$ & $\begin{array}{c}1 \\
1.6 \%\end{array}$ & $\begin{array}{c}7 \\
10.9 \%\end{array}$ & $\begin{array}{c}25 \\
39.1 \%\end{array}$ & $\begin{array}{c}31 \\
48.4 \%\end{array}$ & 0.297 & & \\
\hline 4 & $\begin{array}{l}\text { Through social media, I } \\
\text { can communicate with } \\
\text { anyone at any time }\end{array}$ & $\begin{array}{c}0 \\
0.0 \%\end{array}$ & $\begin{array}{c}0 \\
0.0 \%\end{array}$ & $\begin{array}{c}4 \\
6.3 \%\end{array}$ & $\begin{array}{c}22 \\
34.4 \%\end{array}$ & $\begin{array}{c}38 \\
59.4 \%\end{array}$ & 0.370 & & \\
\hline 5 & $\begin{array}{l}\text { Most of my } \\
\text { communication through } \\
\text { social media is irrelevant } \\
\text { to my studies }\end{array}$ & $\begin{array}{c}0 \\
0.0 \%\end{array}$ & $\begin{array}{c}13 \\
20.3 \%\end{array}$ & $\begin{array}{c}30 \\
46.9 \%\end{array}$ & $\begin{array}{c}15 \\
23.4 \%\end{array}$ & $\begin{array}{c}6 \\
9.4 \%\end{array}$ & 0.270 & & \\
\hline 6 & $\begin{array}{l}\text { Some of my teachers } \\
\text { communicate to me } \\
\text { through social media }\end{array}$ & $\begin{array}{c}2 \\
3.1 \%\end{array}$ & $\begin{array}{c}5 \\
7.8 \%\end{array}$ & $\begin{array}{c}5 \\
7.8 \%\end{array}$ & $\begin{array}{c}38 \\
59.4 \%\end{array}$ & $\begin{array}{c}14 \\
21.9 \%\end{array}$ & 0.359 & & \\
\hline 7 & $\begin{array}{l}\text { I communicate with my } \\
\text { teachers through social } \\
\text { media, but they do not } \\
\text { respond }\end{array}$ & $\begin{array}{c}12 \\
18.8 \%\end{array}$ & $\begin{array}{c}18 \\
28.1 \%\end{array}$ & $\begin{array}{c}15 \\
23.4 \%\end{array}$ & $\begin{array}{c}17 \\
26.6 \%\end{array}$ & $\begin{array}{c}2 \\
3.1 \%\end{array}$ & 0.188 & & \\
\hline 8 & $\begin{array}{l}\text { Communicating through } \\
\text { Social media, I meet } \\
\text { new people }\end{array}$ & $\begin{array}{c}16 \\
25.0 \%\end{array}$ & $\begin{array}{c}11 \\
17.2 \%\end{array}$ & $\begin{array}{c}5 \\
7.8 \%\end{array}$ & $\begin{array}{c}23 \\
35.9 \%\end{array}$ & $\begin{array}{c}9 \\
14.1 \%\end{array}$ & 0.260 & & \\
\hline 9 & $\begin{array}{l}\text { I get to know many } \\
\text { different cultures } \\
\text { through Social media }\end{array}$ & $\begin{array}{c}0 \\
0.0 \%\end{array}$ & $\begin{array}{c}1 \\
1.6 \%\end{array}$ & $\begin{array}{c}8 \\
12.5 \%\end{array}$ & $\begin{array}{c}39 \\
60.9 \%\end{array}$ & $\begin{array}{c}16 \\
25.0 \%\end{array}$ & 0.306 & & \\
\hline 10 & $\begin{array}{l}\text { Social media helps me } \\
\text { to get information about } \\
\text { new job opportunities }\end{array}$ & $\begin{array}{c}0 \\
0.0 \%\end{array}$ & $\begin{array}{c}0 \\
0.0 \%\end{array}$ & $\begin{array}{c}14 \\
21.9 \%\end{array}$ & $\begin{array}{c}39 \\
60.9 \%\end{array}$ & $\begin{array}{c}11 \\
17.2 \%\end{array}$ & 0.311 & & \\
\hline
\end{tabular}


through people I

communicate

The above table shows that the p-value is less than 0.05. So the null hypothesis that 'there is no relationship between the communication of the students and the choices of the respondents' is rejected. i.e., it connotes that there is a significant relationship between the communication of the students and the choices of the respondents. Comparing the K-S values obtained from Kolmogorov-Smirnov test, it is observed that 'Through Social media, I have improved my communication skills' ranks first (0.424) followed by 'Through social media, I can communicate with anyone at any time' (0.370) and 'Some of my teachers communicate to me through social media (0.359).'

Table 5: Relationship

\begin{tabular}{|c|c|c|c|c|c|c|c|c|c|}
\hline \# & Statement & SD & D & $\mathbf{N}$ & $\mathbf{A}$ & SA & $\begin{array}{c}\text { K-S } \\
\text { value }\end{array}$ & $\begin{array}{l}\text { Chi } \\
\text { Square }\end{array}$ & $\begin{array}{c}P \\
\text { value }\end{array}$ \\
\hline 1 & $\begin{array}{l}\text { Using Social media, I have } \\
\text { improved my relationship } \\
\text { with my classmates }\end{array}$ & $\begin{array}{c}1 \\
1.6 \%\end{array}$ & $\begin{array}{c}0 \\
0.0 \%\end{array}$ & $\begin{array}{c}11 \\
17.2 \%\end{array}$ & $\begin{array}{c}33 \\
51.6 \%\end{array}$ & $\begin{array}{c}19 \\
29.7 \%\end{array}$ & 0.273 & \multirow{8}{*}{30.000} & \multirow{8}{*}{.012} \\
\hline 2 & $\begin{array}{l}\text { Through Social media, I } \\
\text { have improved academic } \\
\text { relationship with my } \\
\text { teachers }\end{array}$ & $\begin{array}{c}1 \\
1.6 \%\end{array}$ & $\begin{array}{r}5 \\
7.8 \%\end{array}$ & $\begin{array}{c}15 \\
23.4 \%\end{array}$ & $\begin{array}{c}19 \\
29.7 \%\end{array}$ & $\begin{array}{c}24 \\
37.5 \%\end{array}$ & 0.222 & & \\
\hline 3 & $\begin{array}{l}\text { Social media has improved } \\
\text { my relationships with my } \\
\text { family and friends }\end{array}$ & $\begin{array}{c}2 \\
3.1 \%\end{array}$ & $\begin{array}{c}5 \\
7.8 \%\end{array}$ & $\begin{array}{c}13 \\
20.3 \%\end{array}$ & $\begin{array}{r}29 \\
45.3 \%\end{array}$ & $\begin{array}{c}15 \\
23.4 \%\end{array}$ & 0.274 & & \\
\hline 4 & $\begin{array}{l}\text { I can access my course } \\
\text { material by using social } \\
\text { media tools }\end{array}$ & $\begin{array}{c}1 \\
1.6 \%\end{array}$ & $\begin{array}{c}1 \\
1.6 \%\end{array}$ & $\begin{array}{c}12 \\
18.8 \%\end{array}$ & $\begin{array}{c}39 \\
60.9 \%\end{array}$ & $\begin{array}{c}11 \\
17.2 \%\end{array}$ & 0.331 & & \\
\hline 5 & $\begin{array}{l}\text { Social media helps me to } \\
\text { get knowledge about other } \\
\text { students in different } \\
\text { university and have a } \\
\text { relationship with them }\end{array}$ & $\begin{array}{c}3 \\
4.7 \%\end{array}$ & $\begin{array}{c}0 \\
0.0 \%\end{array}$ & $\begin{array}{c}12 \\
18.8 \%\end{array}$ & $\begin{array}{c}39 \\
60.9 \%\end{array}$ & $\begin{array}{c}10 \\
15.6 \%\end{array}$ & 0.344 & & \\
\hline 6 & $\begin{array}{l}\text { I trust the people whom I } \\
\text { meet on social networking } \\
\text { websites }\end{array}$ & $\begin{array}{c}13 \\
20.3 \%\end{array}$ & $\begin{array}{c}22 \\
34.4 \%\end{array}$ & $\begin{array}{l}23 \\
35.9 \%\end{array}$ & $\begin{array}{c}3 \\
4.7 \%\end{array}$ & $\begin{array}{c}3 \\
4.7 \%\end{array}$ & 0.196 & & \\
\hline 7 & $\begin{array}{l}\text { I spend a lot of time looking } \\
\text { at what other people have } \\
\text { posted }\end{array}$ & $\begin{array}{c}5 \\
7.8 \%\end{array}$ & $\begin{array}{c}10 \\
15.6 \%\end{array}$ & $\begin{array}{c}26 \\
40.6 \%\end{array}$ & $\begin{array}{c}12 \\
18.8 \%\end{array}$ & $\begin{array}{c}11 \\
17.2 \%\end{array}$ & 0.216 & & \\
\hline 8 & $\begin{array}{l}\text { Compared to meeting new } \\
\text { friends in person, I enjoy } \\
\text { more, meeting them online }\end{array}$ & $\begin{array}{c}3 \\
4.7 \%\end{array}$ & $\begin{array}{c}7 \\
10.9 \%\end{array}$ & $\begin{array}{c}17 \\
26.6 \%\end{array}$ & $\begin{array}{c}30 \\
46.9 \%\end{array}$ & $\begin{array}{c}7 \\
10.9 \%\end{array}$ & 0.277 & & \\
\hline
\end{tabular}

The above table shows that the p-value is less than 0.05 . So the null hypothesis that there is no relationship between the Relationship of the students and the choices of the respondents' is rejected. i.e., it connotes that there is a significant relationship between the Relationship of the students and the choices of the respondents. Comparing the K-S values obtained from Kolmogorov-Smirnov test, it is observed that 'Social media helps me to get knowledge about other students in different university and have relationship with them' ranks first (0.344) followed by 'I can access my course material by using social media tools' (0.331) and 'Compared to meeting new friends in person, I enjoy more meeting them online (0.277).'

Table 6: Information reliability

\begin{tabular}{llccccccc}
\hline$\#$ & \multicolumn{1}{c}{ Statement } & SD & D & N & A & SA & $\begin{array}{c}\text { Chi } \\
\text { Square }\end{array}$ & $\begin{array}{c}\text { P } \\
\text { value }\end{array}$ \\
\hline 1 & $\begin{array}{l}\text { I believe all information in } \\
\text { social media is true and } \\
\text { reliable }\end{array}$ & 7 & 12 & 34 & 9 & 2 & 48.031 & .000 \\
\hline 2 & $\begin{array}{l}\text { The quality of the } \\
\text { information in social media }\end{array}$ & $0.0 \%$ & $18.6 \%$ & $53.1 \%$ & $14.1 \%$ & $3.1 \%$ & & \\
\hline
\end{tabular}




\begin{tabular}{|c|c|c|c|c|c|c|c|c|}
\hline & is easily understandable & & & & & & & \\
\hline 3 & $\begin{array}{l}\text { I rely on social media } \\
\text { information }\end{array}$ & $\begin{array}{c}8 \\
12.5 \%\end{array}$ & $\begin{array}{c}13 \\
20.3 \%\end{array}$ & $\begin{array}{c}20 \\
31.3 \%\end{array}$ & $\begin{array}{c}10 \\
15.6 \%\end{array}$ & $\begin{array}{c}13 \\
20.3 \%\end{array}$ & 6.469 & .167 \\
\hline 4 & $\begin{array}{l}\text { The information from social } \\
\text { media helps me to make } \\
\text { better decisions }\end{array}$ & $\begin{array}{c}3 \\
4.7 \%\end{array}$ & $\begin{array}{c}15 \\
23.4 \%\end{array}$ & $\begin{array}{c}11 \\
17.2 \%\end{array}$ & $\begin{array}{c}16 \\
25.0 \%\end{array}$ & $\begin{array}{c}19 \\
29.7 \%\end{array}$ & 11.938 & .018 \\
\hline 5 & $\begin{array}{l}\text { I use information from } \\
\text { social media to solve } \\
\text { complicated issues I face }\end{array}$ & $\begin{array}{c}0 \\
0.0 \%\end{array}$ & $\begin{array}{c}9 \\
14.1 \%\end{array}$ & $\begin{array}{c}22 \\
34.4 \%\end{array}$ & $\begin{array}{c}20 \\
31.3 \%\end{array}$ & $\begin{array}{c}13 \\
20.3 \%\end{array}$ & 6.875 & .076 \\
\hline 6 & $\begin{array}{l}\text { Social media information is } \\
\text { easily available to access at } \\
\text { any point of time }\end{array}$ & $\begin{array}{c}0 \\
0.0 \%\end{array}$ & $\begin{array}{c}0 \\
0.0 \%\end{array}$ & $\begin{array}{c}16 \\
25.0 \%\end{array}$ & $\begin{array}{c}22 \\
34.4 \%\end{array}$ & $\begin{array}{c}26 \\
40.6 \%\end{array}$ & 2.375 & .305 \\
\hline 7 & $\begin{array}{l}\text { Rumours are spread easily } \\
\text { in social media }\end{array}$ & $\begin{array}{c}0 \\
0.0 \%\end{array}$ & $\begin{array}{c}1 \\
1.6 \%\end{array}$ & $\begin{array}{c}11 \\
17.2 \%\end{array}$ & $\begin{array}{c}33 \\
51.6 \%\end{array}$ & $\begin{array}{c}19 \\
29.7 \%\end{array}$ & 34.250 & .000 \\
\hline 8 & $\begin{array}{l}\text { Bad comments are passed } \\
\text { easily through social media } \\
\text { affecting other's sentiments }\end{array}$ & $\begin{array}{c}0 \\
0.0 \%\end{array}$ & $\begin{array}{c}0 \\
0.0 \%\end{array}$ & $\begin{array}{c}10 \\
15.6 \%\end{array}$ & $\begin{array}{c}39 \\
60.9 \%\end{array}$ & $\begin{array}{c}15 \\
23.4 \%\end{array}$ & 22.531 & .000 \\
\hline
\end{tabular}

The p-value for item numbers (3), (5) and (6) in the above table is greater than .05. So after eliminating those factors, we obtained the new table.7 as follows:

Table 7: Information reliability

\begin{tabular}{|c|c|c|c|c|c|c|c|c|c|}
\hline \# & Statement & SD & D & $\mathbf{N}$ & A & SA & $\begin{array}{c}\text { K-S } \\
\text { value }\end{array}$ & $\begin{array}{l}\text { Chi } \\
\text { Square }\end{array}$ & $\begin{array}{c}P \\
\text { value }\end{array}$ \\
\hline 1 & $\begin{array}{l}\text { I believe all information in } \\
\text { social media is true and } \\
\text { reliable }\end{array}$ & $\begin{array}{c}7 \\
10.9 \%\end{array}$ & $\begin{array}{c}12 \\
18.8 \%\end{array}$ & $\begin{array}{c}34 \\
53.1 \%\end{array}$ & $\begin{array}{c}9 \\
14.1 \%\end{array}$ & $\begin{array}{c}2 \\
3.1 \%\end{array}$ & 0.290 & \multirow{5}{*}{27.250} & \multirow{5}{*}{.001} \\
\hline 2 & $\begin{array}{l}\text { The quality of the } \\
\text { information in social } \\
\text { media is easily } \\
\text { understandable }\end{array}$ & $\begin{array}{c}0 \\
0.0 \%\end{array}$ & $\begin{array}{r}1 \\
1.6 \%\end{array}$ & $\begin{array}{l}20 \\
31.3 \%\end{array}$ & $\begin{array}{c}29 \\
45.3 \%\end{array}$ & $\begin{array}{c}14 \\
21.9 \%\end{array}$ & 0.237 & & \\
\hline 4 & $\begin{array}{l}\text { The information from } \\
\text { social media helps me to } \\
\text { make better decisions }\end{array}$ & $\begin{array}{c}3 \\
4.7 \%\end{array}$ & $\begin{array}{c}15 \\
23.4 \%\end{array}$ & $\begin{array}{c}11 \\
17.2 \%\end{array}$ & $\begin{array}{c}16 \\
25.0 \%\end{array}$ & $\begin{array}{c}19 \\
29.7 \%\end{array}$ & 0.195 & & \\
\hline 7 & $\begin{array}{l}\text { Rumours are spread easily } \\
\text { in social media }\end{array}$ & $\begin{array}{c}0 \\
0.0 \%\end{array}$ & $\begin{array}{c}1 \\
1.6 \%\end{array}$ & $\begin{array}{c}11 \\
17.2 \%\end{array}$ & $\begin{array}{c}33 \\
51.6 \%\end{array}$ & $\begin{array}{c}19 \\
29.7 \%\end{array}$ & 0.261 & & \\
\hline 8 & $\begin{array}{l}\text { Bad comments are passed } \\
\text { easily through social media } \\
\text { affecting other's } \\
\text { sentiments }\end{array}$ & $\begin{array}{c}0 \\
0.0 \%\end{array}$ & $\begin{array}{c}0 \\
0.0 \%\end{array}$ & $\begin{array}{c}10 \\
15.6 \%\end{array}$ & $\begin{array}{c}39 \\
60.9 \%\end{array}$ & $\begin{array}{c}15 \\
23.4 \%\end{array}$ & 0.315 & & \\
\hline
\end{tabular}

The above table shows that the p-value is less than 0.05 . So the null hypothesis: "There is no relationship between the Information reliability of the students and the choices of the respondents" is rejected. i.e., it connotes that there is a significant relationship between the information reliability of the students and the choices of the respondents. Comparing the K-S values obtained from Kolmogorov-Smirnov test, it is observed that 'Bad comments are passed easily through social media affecting other's sentiments' ranks first (0.315) followed by 'I believe all information in social media is true and reliable' $(0,290)$ and 'Rumours are spread easily in social media $(0,261)$.'

Table 8: Social Media Effects

\begin{tabular}{cccccccccc}
\hline$\#$ & Statement & SD & D & N & A & SA & $\begin{array}{c}\text { K-S } \\
\text { value }\end{array}$ & $\begin{array}{c}\text { Chi } \\
\text { Square }\end{array}$ & $\begin{array}{c}\text { P } \\
\text { value }\end{array}$
\end{tabular}




\begin{tabular}{|c|c|c|c|c|c|c|c|c|c|}
\hline 1 & $\begin{array}{l}\text { I learn a lot many things } \\
\text { from others through social } \\
\text { media }\end{array}$ & $\begin{array}{c}0 \\
0.0 \%\end{array}$ & $\begin{array}{c}1 \\
1.6 \%\end{array}$ & $\begin{array}{c}16 \\
25.0 \%\end{array}$ & $\begin{array}{c}23 \\
35.9 \%\end{array}$ & $\begin{array}{c}24 \\
37.5 \%\end{array}$ & 0.237 & \multirow{9}{*}{43.844} & \multirow{9}{*}{.000} \\
\hline 2 & $\begin{array}{l}\text { I gain the latest knowledge } \\
\text { about information } \\
\text { technology through social } \\
\text { media }\end{array}$ & $\begin{array}{c}0 \\
0.0 \%\end{array}$ & $\begin{array}{c}3 \\
4.7 \%\end{array}$ & $\begin{array}{c}8 \\
12.5 \%\end{array}$ & $\begin{array}{c}29 \\
45.3 \%\end{array}$ & $\begin{array}{c}24 \\
37.5 \%\end{array}$ & 0.253 & & \\
\hline 3 & $\begin{array}{l}\text { The success of my studies is } \\
\text { based on social media }\end{array}$ & $\begin{array}{c}14 \\
21.9 \%\end{array}$ & $\begin{array}{c}2 \\
3.1 \%\end{array}$ & $\begin{array}{l}17 \\
26.6 \%\end{array}$ & $\begin{array}{c}19 \\
29.7 \%\end{array}$ & $\begin{array}{c}12 \\
18.8 \%\end{array}$ & 0.201 & & \\
\hline 4 & $\begin{array}{l}\text { Social media has badly } \\
\text { affected my curricular } \\
\text { activities }\end{array}$ & $\begin{array}{c}11 \\
17.2 \%\end{array}$ & $\begin{array}{c}18 \\
28.1 \%\end{array}$ & $\begin{array}{c}10 \\
15.6 \%\end{array}$ & $\begin{array}{c}21 \\
32.8 \%\end{array}$ & $\begin{array}{c}4 \\
6.3 \%\end{array}$ & 0.218 & & \\
\hline 5 & $\begin{array}{l}\text { Social media disturbs my } \\
\text { state of mind }\end{array}$ & $\begin{array}{c}2 \\
3.1 \%\end{array}$ & $\begin{array}{c}21 \\
32.8 \%\end{array}$ & $\begin{array}{c}16 \\
25.0 \%\end{array}$ & $\begin{array}{c}21 \\
32.8 \%\end{array}$ & $\begin{array}{c}4 \\
6.3 \%\end{array}$ & 0.211 & & \\
\hline 6 & $\begin{array}{l}\text { Curious to know the latest } \\
\text { information in the social } \\
\text { media }\end{array}$ & $\begin{array}{c}1 \\
1.6 \%\end{array}$ & $\begin{array}{c}8 \\
12.5 \%\end{array}$ & $\begin{array}{c}14 \\
21.9 \%\end{array}$ & $\begin{array}{c}32 \\
50.0 \%\end{array}$ & $\begin{array}{c}9 \\
14.1 \%\end{array}$ & 0.297 & & \\
\hline 7 & $\begin{array}{l}\text { During exam time, I believe } \\
\text { that someone will leak the } \\
\text { exam paper in social media }\end{array}$ & $\begin{array}{c}8 \\
12.5 \%\end{array}$ & $\begin{array}{c}21 \\
32.8 \%\end{array}$ & $\begin{array}{c}10 \\
15.6 \%\end{array}$ & $\begin{array}{c}19 \\
29.7 \%\end{array}$ & $\begin{array}{c}6 \\
9.4 \%\end{array}$ & 0.222 & & \\
\hline 8 & $\begin{array}{l}\text { Social media message tone } \\
\text { diverts my attention from } \\
\text { my work }\end{array}$ & $\begin{array}{c}2 \\
3.1 \%\end{array}$ & $\begin{array}{c}11 \\
17.2 \%\end{array}$ & $\begin{array}{c}18 \\
28.1 \%\end{array}$ & $\begin{array}{c}29 \\
45.3 \%\end{array}$ & $\begin{array}{c}4 \\
6.3 \%\end{array}$ & 0.272 & & \\
\hline 9 & $\begin{array}{l}\text { Social media gives a proud } \\
\text { feeling that I can use social } \\
\text { media }\end{array}$ & $\begin{array}{c}1 \\
1.6 \%\end{array}$ & $\begin{array}{c}18 \\
28.1 \%\end{array}$ & $\begin{array}{c}23 \\
35.9 \%\end{array}$ & $\begin{array}{c}11 \\
17.2 \%\end{array}$ & $\begin{array}{c}11 \\
17.2 \%\end{array}$ & 0.230 & & \\
\hline
\end{tabular}

The above table shows that the p-value is less than 0.05. So the null hypothesis: "There is no relationship between the Social Media Effects of the students and the choices of the respondents" is rejected. i.e., it connotes that there is a significant relationship between the Social Media effects of the students and the choices of the respondents. Comparing the K-S values obtained from Kolmogorov-Smirnov test, it is observed that 'Curious to know the latest information through social media' ranks first (0.297) followed by 'Social media message tone diverts my attention from my work' $(., 272)$ and 'I gain the latest knowledge about information technology through social media $(0.253)$.'

Table 9: (a), (b), (c) \& (d) Regression

Variables Entered / Removed

\begin{tabular}{llcc}
\hline Model & Variables Entered & $\begin{array}{l}\text { Variables } \\
\text { Removed }\end{array}$ & Method \\
\hline 1 & Social media effects, & $\ldots$ & Enter \\
& $\begin{array}{l}\text { Information reliability, } \\
\text { Communication, }\end{array}$ & & \\
& Relationship & & \\
\hline
\end{tabular}

${ }^{a}$ Dependent Variable: Learning

${ }^{\mathrm{b}}$ All requested Variables entered.

Model Summary

\begin{tabular}{lcccc}
\hline Model & $\mathrm{R}$ & R Square & $\begin{array}{l}\text { Adjusted R } \\
\text { Square }\end{array}$ & $\begin{array}{l}\text { Std. The error of } \\
\text { the Estimate }\end{array}$ \\
\hline 1 & .447 & .200 & .145 & 2.84603 \\
\hline
\end{tabular}

${ }^{a}$ Predictors: (constant), Information reliability, Social media effect, Communication, Relationship

\section{ANOVA $^{\mathrm{a}}$}




\begin{tabular}{|c|c|c|c|c|c|}
\hline Model & $\begin{array}{l}\text { Sum Of } \\
\text { Squares }\end{array}$ & df & Mean Square & $\mathrm{F}$ & Sig. \\
\hline Regression & 119.218 & 4 & 29.804 & 3.680 & $.010^{\mathrm{b}}$ \\
\hline Residual & 477.892 & 59 & 8.100 & & \\
\hline Total & 597.109 & 63 & & & \\
\hline
\end{tabular}

${ }^{\mathrm{a}}$ Dependent Variable: Learning

${ }^{\mathrm{b}}$ Predictors: (constant), Information reliability, Social media effects, Communication, Relationship.

Coefficients

\begin{tabular}{lccccc}
\hline \multicolumn{1}{c}{ Model } & \multicolumn{2}{c}{ Unstandardized Coefficients } & $\begin{array}{c}\text { Standardized } \\
\text { Coefficients }\end{array}$ & $\mathrm{t}$ & Sig. \\
\hline & $\mathrm{B}$ & Std.Error & Beta & & \\
\hline (constant) & 18.558 & 3.639 & & 5.099 & .000 \\
\hline Communication & .055 & .117 & .067 & 0.473 & .638 \\
\hline Relationship & .083 & .108 & .119 & 0.770 & .445 \\
\hline Information reliability & .205 & .217 & .173 & 0.944 & .349 \\
\hline Social Media Effects & .116 & .107 & .178 & 1.083 & .283 \\
\hline
\end{tabular}

${ }^{\mathrm{a} D e p e n d e n t ~ V a r i a b l e: ~ L e a r n i n g ~}$

From the above table, it can be seen that the p-value for all the dependent variables is more than 0.05. i.e. Learning is not dependent on any of the independent variables. In other words, Communication through Social Media, Relationships earned through social media, Information reliability of Social Media and the Social media effects have no impact on the Learning. Thus, it is confirmed that Social Media does not support the Learning of the students.

\section{CONCLUSION AND SUGGESTIONS}

From the above, it is observed that students exchange learning materials through social media and it helps them to update the developments in their college/university. Their teachers also encourage them to use social media but to study. The students believe that through Social media they have improved their communication skills and they can communicate with anyone at any time. It is also found that some of their teachers communicate with them through social media.

Most of the students reported that social media help them to attain knowledge about other students in different universities and have a relationship with them and they can access their course materials by using social media tools.

Further, they reported that they enjoy meeting new friends online using social media rather than meeting in person and for this reason they spend a lot of time - addicted. It is also confirmed by most of the respondents that the bad comments are passed easily through social media affecting other's sentiments and most of them strongly believe that all the information in social media is true and reliable and they also agreed that rumors were spread easily in social media.

Also, through the regression analysis, it is confirmed that social media does not support the Learning of the students.

\section{RECOMMENDATIONS}

Students could have better control over social media use if and only if they have more self-regulation (Rouis, Limayem, \& Salehi-Sangari, 2011b).

The following recommendations will guide to draw an effective social media strategy:

1. The stringent policy should be deployed in HEIs on the students' internet usage.

2. Excessive time spending on social media should be curtailed.

3. Educational programs on proper sleeping habits, social media hygiene, and recovery of weekend sleep debt should be considered (Al Suwayri, 2016).

4. Cyberbullying and pornography should not be permitted.

5. The education-related information only should be made available to the students.

6. There are dangerous communities that exist in Social Media sites targeting mainly the teens and adults as the students are liable to disclose their personal information which might harm them personally and hence they should be properly advised. (El Khatib \& Khan, 2017).

7. Effective technical monitoring should be made while students browse these sites and their activities may be kept under surveillance.

8. Last but not least, parents should proactively involve in monitoring their children's activities. 


\section{REFERENCES}

1. Al-Tarawneh, H. A. (2014). The influence of social networks on students' performance. Journal of Emerging Trends in Computing and Information Sciences, 5(3), 200-205.

2. Al Suwayri, S. M. (2016). The impact of social media volume and addiction on medical student sleep quality and academic performance: a cross-sectional observational study. Imam Journal of Applied Sciences, 1(2), 80-87. doi: 10.4103/ijas.ijas_34_16

3. Ali Aljabry, A. M., Ahmed Jaafari, A. A., Salawi, M., Abdullah, M., Majrabi, T., Abdoh, F., .. Daghriri, M. (2017). Effect of Social Media Network on Social Relations and Academic Achievement Among Medical Students. Egyptian Journal of Hospital Medicine, 69(7), 2910-2917. https://doi.org/10.12816/0042585

4. Allam, M., \& Elyas, T. (2016). Perceptions of Using Social Media as an ELT Tool among EFL Teachers in the Saudi Context. English Language Teaching, 9(7), 1-9. https://doi.org/10.5539/elt.v9n7p1

5. Alsuraihi, A. K., Almaqati, A. S., Abughanim, S. A., \& Jastaniah, N. A. (2016). Use of Social Media in Education among Medical Students in Saudi Arabia. Korean journal of medical education, 28(4), 343-354. https://doi.org/10.3946/kjme.2016.40

6. Alwagait, E., Shahzad, B., \& Alim, S. (2015). Impact of social media usage on students academic performance in Saudi Arabia. Computers in Human Behavior, 51(Part B), 1092-1097. ttps://doi.org/10.1016/j.chb.2014.09.028

7. Amadi, E. C., \& Ewa, C. (2018). Effects of social media on the academic performance of University students in Nigeria: A study of the Rivers State University, Port Harcourt. International Journal of Innovative Information Systems \& Technology Research, 6(1), 21-27.

8. Amin, Z., Mansoor, A., Hussain, S. R., \& Hashmat, F. (2016). Impact of Social Media of Student's Academic Performance. International Journal of Business and Management Invention, 5(4), 22-29.

9. Baker, B. (2009). Your customer is talking-to everyone. Information management, 19(4), 20.

10. Boyd, D. (2007a). Social network sites: Public, private, or what. Knowledge Tree, 13(1), 1-7.

11. Boyd, D. (2007b). Why Youth (heart) Social Network Sites: The Role of Networked Publics in Teenage Social Life. In D. Buckingham (Ed.), MacArthur foundation series on digital learning-Youth, identity, and digital media volume (Vol. Research Publication No.2007-16, pp. 119-142). Cambridge, MA: MIT Press. https://doi.org/10.31219/osf.io/22hq2

12. Butler, B. S., \& Matook, S. (2015). Social Media and Relationships. In P.H.Ang \& R. Mansell (Eds.), The International Encyclopedia of Digital Communication and Society (pp. 1-12). Retrieved from https://doi.org/10.1002/9781118767771.wbiedcs097. https://doi.org/10.1002/9781118767771.wbiedcs097

13. Chretien, K. C., Farnan, J. M., Greysen, S. R., \& Kind, T. (2011). To Friend or Not to Friend? Social Networking and Faculty Perceptions of Online Professionalism. Academic Medicine, 86(12), 1545-1550. https://doi.org/10.1097/ACM.0b013e3182356128

14. El-Badawy, T. A., \& Hashem, Y. (2015). The Impact of Social Media on the Academic Development of School Students. International Journal of Business Administration, 6(1), 46. https://doi.org/10.5430/ijba.v6n1p46

15. El Khatib, M., \& Khan, F. R. (2017). Implications of Social Media Networks Technology in Interpersonal Skills and Academic Performances. International Journal of Management, Innovation \& Entrepreneurial Research, 3(2), 99110. https://doi.org/10.18510/ijmier.2017.326

16. Ellore, S. B., Niranjan, S., \& Brown, U. J. (2014). The influence of internet usage on academic performance and faceto-face communication. Journal of Psychology and Behavioral Science, 2(2), 163-186.

17. Greysen, S. R., Kind, T., \& Chretien, K. C. (2010). Online professionalism and the mirror of social media. Journal of general internal medicine, 25(11), 1227-1229. https://doi.org/10.1007/s11606-010-1447-1

18. Griffiths, M. D. (2013). Social networking addiction: Emerging themes and issues. Journal of Addiction Research \& Therapy, 4(5), 1-2. doi: 10.4172/2155-6105.1000e118

19. Halboub, E., Othathi, F., Mutawwam, F., Madkhali, S., Somaili, D., \& Alahmar, N. (2016). Effect of social networking on academic achievement of dental students, Jazan University, Saudi Arabia. EMHJ-Eastern Mediterranean Health Journal, 22(12), 865-871. https://doi.org/10.26719/2016.22.12.865

20. Hasnain, H., Nasreen, A., \& Ijaz, H. (2015). Impact of social media usage on academic performance of university students. Paper presented at the 2nd International Research Management \& Innovation Conference (IRMIC).

21. Jameel, T., Gazzaz, Z. J., Baig, M., Tashkandi, J. M., Alharenth, N. S., Butt, N. S., . . Iftikhar, R. (2019). Medical students' preferences towards learning resources and their study habits at King Abdulaziz University, Jeddah, Saudi Arabia. BMC research notes, 12(1), 30. https://doi.org/10.1186/s13104-019-4052-3

22. Junco, R. (2015). Student class standing, Facebook use, and academic performance. Journal of Applied Developmental Psychology, 36, 18-29. https://doi.org/10.1016/j.appdev.2014.11.001

23. Karpinski, A. C., Kirschner, P. A., Ozer, I., Mellott, J. A., \& Ochwo, P. (2013). An exploration of social networking site use, multitasking, and academic performance among United States and European university students. Computers in Human Behavior, 29(3), 1182-1192. https://doi.org/10.1016/j.chb.2012.10.011 
24. Khan, F. R., Al-balushi, H., Algaithi, A., \& Al-Shihi, A. (2017). Impact of Social Media on Customers Satisfaction: Bank Muscat-A Case Study. Ahead International Journal of Recent Research Review, 1(11), 154-163.

25. Khan, F. R., Hatami, Y., Sasidharan, A., \& Al-Roshdi, S. (2017). Investigative Study of Preferred Social Media Marketing in Safeer Mall, Sohar, Oman. Humanities \& Social Science Reviews, 5(1), 53-63. ttps://doi.org/10.18510/hssr.2017.515

26. Lomi, A., Snijders, T. A., Steglich, C. E., \& Torló, V. J. (2011). Why are some more peer than others? Evidence from a longitudinal study of social networks and individual academic performance. Social Science Research, 40(6), 15061520. https://doi.org/10.1016/j.ssresearch.2011.06.010

27. Masters, K. (2015). Social networking addiction among health sciences students in Oman. Sultan Qaboos University Medical Journal, 15(3), 357-363. dhttps://doi.org/10.18295/squmj.2015.15.03.009

28. Mehmood, S., \& Taswir, T. (2013). The effects of social networking sites on the academic performance of students in college of applied sciences, Nizwa, Oman. International Journal of Arts and Commerce, 2(1), 111-125.

29. Michikyan, M., Subrahmanyam, K., \& Dennis, J. (2015). Facebook use and academic performance among college students: A mixed-methods study with a multi-ethnic sample. Computers in Human Behavior, 45, 265-272. https://doi.org/10.1016/j.chb.2014.12.033

30. Mingle, J., \& Adams, M. (2015). Social Media Network Participation and Academic Performance in Senior High Schools in Ghana. Library Philosophy and Practice(Paper 1286), 1-51.

31. Osharive, P. (2015). Social Media and Academic Performance of Students. (Degree in Educational Administration Bachelor Degree Research Project), Faculty of Education, University of Lagos, Lagos. Retrieved from https://www.researchgate.net/publication/273765340_social_media_and_academic_performance_of_students $(100302125)$

32. Owusu-Acheaw, M., \& Larson, A. G. (2015). Use of Social Media and Its Impact on Academic Performance of Tertiary Institution Students: A Study of Students of Koforidua Polytechnic, Ghana. Journal of Education and Practice, 6(6), 94-101.

33. Paul, J. A., Baker, H. M., \& Cochran, J. D. (2012). Effect of online social networking on student academic performance. Computers in Human Behavior, 28(6), 2117-2127. https://doi.org/10.1016/j.chb.2012.10.011

34. Rouis, S., Limayem, M., \& Salehi-Sangari, E. (2011a). Impact of Facebook Usage on Students Academic Achievement: Role of self-regulation and trust. Electronic Journal of Research in Educational Psychology, 9(3), 961994. https://doi.org/10.25115/ejrep.v9i25.1465

35. Rouis, S., Limayem, M., \& Salehi-Sangari, E. (2011b). Impact of Facebook Usage on Students Academic Achievement: Role of self-regulation and trust.

36. Squmedia-ar. (2015). Health Students Addicted to Social Net. Retrieved 10th Oct. 2019, from Sultan Qaboos University https://www.squ.edu.om/squmedia/Details-Page/ArticleID/2969/Health-Science-Students-Addicted-toSocial-Net

37. Wentworth, D. K., \& Middleton, J. H. (2014). Technology use and academic performance. Computers \& Education, 78, 306-311. https://doi.org/10.1016/j.compedu.2014.06.012

38. Yusuf, N., AL-Madah, R., \& Alam, M. Z. (2016). Social media as a tool in learning and social behavior in Saudi Arabia. International Journal of Higher Education Management, 3(1), 65-74. 